\title{
DIETER GOSEWINKEL
}

\section{INTRODUCTION II \\ HISTOIRE ET HISTORIOGRAPHIE DE L'ÉTAT EN FRANCE ET EN ALLEMAGNE*}

Peu de concepts ont autant relié l'univers des idées politiques et l'histoire de la France et de l'Allemagne que l'»État«. La conception d'un État stable, hiérarchisé et aux rouages bien huilés, selon le modèle de l'absolutisme français, a exercé une longue attraction et a servi durablement de modèle sous le Saint Empire romain germanique, comme il a influencé le développement de l'État territorial de l'Empire allemand. Les deux pays ont conféré à l'État une signification centrale, pour ainsi dire mythique, dans l'émergence, la légitimation et la stabilisation de l'ordre politique. Mais si le concept d'»État« est important dans les deux pays, il y a prédominé à des époques différentes. Alors qu'après 1789 l'hégémonie du concept "État" faiblit en France, elle s'établit dans la langue politico-sociale de l'Allemagne. En France, les termes de srépublique» et d'»empire« passent alors au premier plan, et ce n'est qu'après 1800 qu'un État allemand, précisément un État-nation transcendant les États territoriaux, devient l'objectif politique central. Il n'est possible de parler d'un `État allemand que depuis la césure de 1870/71, point initial des recherches de cet ouvrage. Ce rretard de l'État allemand peut contribuer à expliquer pourquoi les historiographies des deux États ont réagi différemment aux efforts de démystification de l'État, à son éviction par des approches interprétatives sociohistoriques, ainsi qu'à sa déconstruction historico-culturelle. En France, on a en somme porté moins d'attention à l'État, tant son existence et sa signification étaient assurées. En Allemagne en revanche, il est toujours resté l'objet de l'intérêt historiographique, du fait même de la précarité de son existence et de sa fragilité politique.

Les efforts visant à créer un État (centralisé) en Allemagne, l'expansion du pouvoir, la destruction et la segmentation de cet État entre 1870 et 1945 , reflètent un mouvement politique incessant qui s'accompagne d'une démultiplication du champ notionnel. Au terme "Staat" (État avec un grand E), la langue allemande accole une multitude de dérivés et de distinctions sémantiques pour spécifier l'État: "Rechtsstaat « (État de droit), "Kulturstaat« (État culturel), "Machtstaat" (État de force), "Nationalstaat« (État-nation), "Wirtschaftsstaat« (État économique) »Volksstaat« (État populaire) »Sozialstaat» (État social) et enfin le »tota-

\footnotetext{
* Traduit de l'allemand par Valentine Meunier.
} 
ler Staat«, l'État total 'du national-socialisme ${ }^{1}$. Cette diversité reflète d'une part l'effort continuel pour s'assurer de l'existence de l'État sous toutes ses facettes, et traduit d'autre part la conception d'un État omniprésent, qui intervient dans tous les domaines en les structurant et en les dirigeant.

Traditions et nouvelles approches de l'historiographie allemande sur l'État

La continuité de la "science politique (Staatswissenschaft) allemande, de la science de l'État en tant qu'axe de recherche fondamentalement interdisciplinaire ${ }^{2}$ s'incarne dans la revue "Der Staat«, avec son sous-titre "Revue de la théorie de l'État et de l'histoire constitutionnelle, de droit public allemand et européen «. Fondée en 1962 par Gerhard Oestreich, Ernst Forsthoff et Hans Barion, cette revue perpétue la tradition de combiner des travaux de théorie politique, historiques et constitutionnalistes avec l'objet "État«. D'un point de vue de l'histoire des sciences ${ }^{3}$, il est possible de tracer des lignes de continuité partant de cette revue et menant à l'ouverture disciplinaire et méthodologique de l'analyse scientifique de l'État depuis le début du $\mathrm{XX}^{\mathrm{e}}$ siècle. Avec l'affaiblissement du positivisme en droit public, l'essor de la sociologie historique de l'État chez Max Weber et Georg Jellinek, l'histoire constitutionnelle comparative de Otto Hintze, et finalement avec l'ouverture de la science juridique publiciste à la philosophie, à la théologie et à la science politique balbutiante, grâce à des personnalités telles que Rudolf Smend, Carl Schmitt et Hermann Heller, émergea sous la République de Weimar une science de l'État, qui prit au sérieux les principes de l'histoire des idées et de la sociologie et servit à interpréter les institutions de droit public. Les ouvrages de Carl Schmitt tout particulièrement, dont la réception, transmise pour l'essentiel par la théorie institutionnelle de Maurice Hauriou, influença également la science française de l'État. Les écrits de Schmitt sont ceux qui ont le plus profondément marqué le débat autour de "l'État« dans la revue et plus largement. Constamment réédités et maintes fois traduits, ses écrits sur l'État ont beaucoup d'influence dans de nombreux pays d'Europe. L'œuvre de Schmitt divise le débat scientifique plus que celle de tout

' Reinhart KOSELLECK, Préface à l'article Staat und Souveränität, dans: Geschichtliche Grundbegriffe, t. 6, Stuttgart 1990, p. 1-4 (3).

${ }^{2}$ Cf. aussi la revue »Zeitschrift für die gesamte Staatswissenschaft«, qui paraît depuis 1844 (à partir de 1985 sous le titre »Journal of institutional and theoretical economics«).

${ }^{3}$ Sur la science allemande de l'État au XIX ${ }^{\mathrm{e}}$ siècle, cf. David F. LJNDENFELD, The Practical Imagination. The German Sciences of State in the Nineteenth Century, Chicago, Londres 1997. 
autre publiciste allemand du $\mathrm{XX}^{\mathrm{e}}$ siècle ${ }^{4}$. Ernst-Wolfgang Böckenförde, qui a durablement influencé la revue depuis sa création, incarne d'une manière toute particulière la vaste ambition scientifique qui entend saisir les fondements théoriques et philosophiques; historiques et constitutionnels ainsi que les références théologiques de l'État. Les ouvrages de Böckenförde ${ }^{5}$ vont de la théorie de l'État antique à la théorie démocratique de la Loi fondamentale. Ils analysent l'État du constitutionnalisme allemand, de la République de Weimar et du national-socialisme tout autant que les structures fondamentales de »l'association d'États « - le Staatenverbund selon le terme de la Cour constitutionnelle fédérale allemande - que forme l'Union européenne. Böckenförde personnifie une spécificité de ll'étaticité allemande après 1945, à la croisée de la science: l'extraordinaire importance de la juridiction constitutionnelle et donc des chercheurs en droit public, qui peuvent être nommés juges à la Cour constitutionnelle fédérale et de là peser sur la jurisprudence de la plus haute juridiction allemande par leurs positions scientifiques. Ernst-Wolfgang Böckenförde, Dieter Grimm $^{6}$ et Paul Kirchhof ${ }^{7}$, par exemple, font partie des publicistes qui, en leur qualité de juges à la Cour constitutionnelle, ont conjointement influé sur l'interprétation du droit constitutionnel en vigueur grâce à leurs ouvrages théoriques et historiques.

Si ce débat sur l'État est traditionnellement modelé par des juristes intimement liés à l'État par leur disćipline scientifique et leur pratique professionnelle, on relève depuis les années 1980 une tendance croissante des sciences sociales à réintégrer l'État comme un objet d'analyse légitime et digne d'intérêt dans leur

${ }^{4}$ Après 1945, une vaste littérature a paru sur Carl SCHMITT, rassemblée par des membres de l'école de Schmitt sous le titre "Schmittiana«. Outre les biographies, il faut notamment mentionner les ouvrages qui, recourant aux méthodes d'une sintellectual history«, placent Schmitt au centre d'un réseau intellectuel. Dans cette perspective cf. en particulier Dirk van LAAK, Gespräche in der Sicherheit des Schweigens. Carl Schmitt in der politischen Geistesgeschichte der frühen Bundesrepublik, Berlin ${ }^{2} 2002$; par ailleurs, ID., Glossarium. Aufzeichnungen der Jahre 1947-1951, Berlin 1991.

${ }^{5}$ Ernst-Wolfgang BÖCKENFÖRDE, Gesetz und gesetzgebende Gewalt: Von den Anfängen der deutschen Staatsrechtslehre bis zur Höhe des staatsrechtlichen Positivismus, Berlin ${ }^{2} 1981$; ID., Geschichte der Rechts- und Staatsphilosophie: Antike und Mittelalter, Tübingen 2002; ID., Staat, Gesellschaft, Freiheit: Studien zur Staatstheorie und zum Verfassungsrecht, Francfort/M. 1976; ID., Recht, Staat, Freiheit. Studien zur Rechtsphilosophie, Staatstheorie und Verfassungsgeschichte, Francfort/M. 1991; ID., Staat, Verfassung, Demokratie. Studien zur Verfassungstheorie und zum Verfassungsrecht, Francfort/M. 1991; ID., Staat, Nation, Europa. Studien zur Staatslehre, Verfassungstheorie und Rechtsphilosophie, Francfort/M. 1999.

${ }^{6}$ Dieter GRIMM, Deutsche Verfassungsgeschichte 1776-1866. Vom Beginn des modernen Verfassungsstaats bis zur Auflösung des Deutschen Bundes, Francfort/M. ${ }^{3} 1995$; ID., Recht und Staat der bürgerlichen Gesellschaft, Francfort/M. 1987; ID., Zukunft der Verfassung, Francfort/M. 1991.

${ }^{7}$ Paul KIRCHHOF tout particulièrement grâce à l'ouvrage fondamental qu'il a dirigé avec Josef ISENSEE, Handbuch des Staatsrechts der Bundesrepublik Deutschland, 10 vols, Heidelberg 1987-2000. 
débat. Sous la devise "Bringing the State Back in«, Peter B. Evans, Dietrich Rueschemeyer et Theda Skocpol, chercheurs américains en sciences sociales, ont montré que la problématique de l'État, compris comme acteur et institution structurant la société, avait connu une "envolée« à partir du milieu des années 1970 dans la littérature des sciences sociales. Ils rappelèrent la longue tradition des recherches sur l'État moderne, d'Alexis de Tocqueville, Max Weber et Otto Hintze aux marxistes de toutes tendances, même si ces derniers adoptent une attitude critique à l'égard de l'État. Ils firent remarquer que l'État wavait [longtemps] été considéré comme un concept obsolète, lié à des recherches juridiques formalistes austères et poussiéreuses, se consacrant aux particularismes nationaux des principes constitutionnels ${ }^{8}$. Mais c'était précisément ce qui était en train de changer: de plus en plus de travaux historiques et comparatifs étaient entrepris, qui considéraient l'État comme un facteur »autonome« de la politique, doté d'un véritable pouvoir de forger la société. Ces travaux pensaient l'État dans une perspective macrosociologique comme wune configuration de l'organisation et de l'action« influençant »la signification et les méthodes de la politique pour tous les groupes et classes de la société«?.

Il existe de multiples raisons expliquant la résurgence de l'intérêt, depuis lors ininterrompu, pour l'État et pour son histoire. Nous en retiendrons deux ici. Première raison: le revirement méthodologique que les sciences humaines et sociales ont connu depuis la fin des années 1970. Les sciences humaines et sociales intégrèrent certaines méthodes des "sciences de la culture" (Kulturwissenschaft). Le "processus de la civilisation" étayé par les théories de Norbert Elias $^{10}$, accrût l'intérêt porté à l'État dans l'histoire culturelle. Conforté par les influences de l'anthropologie sociale ${ }^{11}$, on s'écartait de l'analyse institutionnelle pour se concentrer sur les formes de représentation de l'État, sur les modèles et

\footnotetext{
${ }^{8}$ Theda SKOCPOL, Bringing the State Back in: Strategies of Analysis in Current Research, dans: ID., Peter B. EvanS, Dietrich RUESCHEMEYER (dir.), Bringing the State back in, Cambridge 1985 , p. 4 .

${ }^{9}$ Ibid. p. 28.

${ }^{10}$ Norbert Elias, Was ist Soziologie?, München ${ }^{6} 1991$, p.139-140; ID., Studien über die Deutschen. Machtkämpfe und Habitusentwicklung im 19. und 20. Jahrhundert, Francfort/M. 1992.

"Alexandra PRZYREMBEL, "Rassenschande«: Sexualität, »Rasse« und das »Jüdische« vor NS-Gerichten in den Jahren 1935 bis 1945, dans: Historische Anthropologie 12 (2004) p. 338-354; Jürgen MARTSCHUKAT, Der »Maßstab für die geistige Bildungsstufe eines Volkes und die Moralität eines Zeitalters «. Die Todesstrafe in Diskurs und Praxis im 18. und 19. Jahrhundert, dans: Historische Anthropologie 9 (2001) p. 1-26; Dorothee WIERLiNG, Über die Liebe zum Staat - der Fall der DDR, dans: Historische Anthropologie 8 (2000) p. 236-263; Christiane EIFERT, Die kleinen Könige. Zu Selbstverständnis und Herrschaftspraxis brandenburgischer Landräte im 19. Jahrhundert, dans: Historische Anthropologie 7 (1999) p. 381-403; pour un exemple en histoire contemporaine: Edouard CONTE, Cornelia ESSNER, La Quête de la Race. Une anthropologie du nazisme, Paris 1995.
} 
les symboles du pouvoir ${ }^{12}$. Elias contribua aussi à définir la notion de $»$ figuration«, entendue comme instrument pour surmonter l'antagonisme normatif entre la société et l'individu, dont le titre de ce volume »Figures de l'État - Figurationen des Staates « porte la trace.

Parallèlement à la croissance des méthodes et des objets des sciences de la culture, l'histoire et l'analyse structurelle de la société perdit l'hégémonie qu'elle avait acquise entre la fin des années 1950 à la fin des années 1970. La société en tant que paradigme central de la recherche en histoire et en sciences sociales perdit son statut avant-gardiste. Ce fut la fin du triomphe de l'histoire sociale entendue comme une méthode dans laquelle l'État constituait un facteur historique parmi d'autres, souvent interprété par rapport à la société et à ses forces actives ${ }^{13}$. En abandonnant son statut avant-gardiste et, dans un champ scientifique de plus en plus défini par le pluralisme méthodologique, l'histoire sociale laissa place à des recherches qui une ou deux décennies auparavant auraient encore été taxées d'étatisme anachronique. Dans la foulée d'une »nouvelle histoire politique ${ }^{14}$, des travaux historiques émergèrent, qui ne se contentaient pas de postuler l'État comme une unité de mesure, mais l'interrogeaient comme une entité, une structure historiquement variable d'organisation et d'institutions et qui en étudiaient le fonctionnement social.

Seconde raison de la renaissance de l'État en sciences humaines et sociales: les changements structurels, et pour certains radicaux, qui se produisirent dans la sphère de l'expérience politique. Les bouleversements révolutionnaires en Europe de l'Est, la rapidité époustouflante de la chute des systèmes étatiques centralisés que l'on croyait fermement structurés, la formation nouvelle, accélérée et souvent violente, du système étatique en Europe centrale et orientale, sont autant d'événements qui avivèrent l'intérêt pour l'émergence, la fonction et les modes de fonctionnement des États, ainsi que l'indique la multitude de recherches sur la transformation ${ }^{15}$ des États post-communistes. Parallèlement aux bouleversements

${ }^{12}$ Les travaux de l'historien de l'art Horst Bredekamp en constituent un excellent exemple, en particulier Horst BREDEKAMP, Thomas Hobbes. Der Leviathan: das Urbild des modernen Staates und seine Gegenbilder 1651-2001, Berlin ${ }^{2} 2003$.

${ }^{13}$ Jürgen KOCKA, Sozialgeschichte. Begriff, Entwicklung, Probleme, Göttingen 1977; HansUlrich WEHLER, Vom Feudalismus des Alten Reiches bis zur Defensiven Modernisierung der Reformära, 1700-1815. Deutsche Gesellschaftsgeschichte t. 1, Munich 1987.

${ }^{14}$ Ute FREVERT, Heinz-Gerhard HAUPT, Neue Politikgeschichte. Perspektiven einer historischen Politikforschung, Francfort/M. 2005; Michael MAURER, Neue Themen und Methoden der Geschichtswissenschaft, Stuttgart 2003.

${ }^{15}$ Günther HEYDEMANN, Gunther MAI, Werner MüLleR (dir.), Revolution und Transformation in der DDR 1989/90, Berlin 1999; Helmut WIESENTHAL, Die Transformation der DDR. Verfahren und Resultate, Gütersloh 1999; ID. (dir.), Einheit als Privileg. Vergleichende Perspektiven auf die Transformation Ostdeutschlands, Francfort/M. 1996; Christof EHRHART, Transformation in Ungarn und in der DDR. Eine vergleichende Analyse, Opladen 1998; Ellen Bos, Verfassungsgebung und Systemwechsel. Die Institutionalisierung von Demokratie im postsozialistischen Osteuropa, Wiesbaden 2004; Sten BERGLUND, Frank H. 
externes des États, l'attention se porta progressivement sur les profondes transformations structurelles internes de l'État '(post)moderne`. Ce furent désormais les nouvelles formes gouvernementales et administratives, par-delà les fonctions traditionnelles du pouvoir et de la souveraineté, qui formèrent le coeur des analyses. L'État coopératif, sdégraissé< et décentralisé tint lieu de modèle à un nouveau courant de recherche, qui rassembla des chercheurs en droit et en sciences socia${ }^{\text {les }}{ }^{16}$ de toutes disciplines. Le "govemment«, objet de recherche décrié, laissa place à un nouveau paradigme bien plus au goût du jour, la "governance«. Ces recherches ont également pour partie recours à des arguments historiques et dessinent des lignes d'évolution historiques qui mènent à l'État coopératif. Il en va de même pour une troisième évolution qui modifie profondément l'ordre public contemporain: l'implantation de formes d'organisation et d'institutions trans- et supranationales. L'organisation qu'est l'Union européenne, entre État et superÉtat, que le droit public peine à catégoriser, et les multiples brèches transnationales dans la souveraineté étatique (nationale) ont stimulé la recherche de parallèles et de modèles historiques. Dès lors, l'attention s'est déportée sur la dite première phase de globalisation au toumant $\mathrm{du} \mathrm{XIX}^{\mathrm{e}}$ au $\mathrm{XX}^{\mathrm{e}}$ siècle ${ }^{17}$, sur les formes d'organisations internationales de l'entre-deux-guerres ou sur une période plus ancienne encore, la constellation étatique du Saint Empire romain germanique ${ }^{18}$.

\section{Le champ thématique: du droit public à l'État colonial}

La science juridique joue un rôle central dans l'historiographie allemande sur l'État. En effet, le concept allemand de l'État a ceci de spécifique qu'il est un concept juridique. C'est l'hypothèse que démontre Christoph Möllers pour

AAREBROT, Henri VOGT et al., Challenges to Democracy. Eastern Europe ten years after the collaps of communism, Celtenham 2001; David S. MASON, James R. KLUEGEL, Ludmila KHAKHULINA, Marketing democracy. Changing opinion about inequality and politics in East Central Europe, Lanham 2000.

${ }^{16}$ Une recherche d'ores et déjà incontournable, par ex. Richard BELLAMY, Alex WARLEIGH (dir.), Citizenship and governance in the European Union, Londres 2001; de premier ordre dans le domaine de la science juridique: Gunnar Folke SCHUPPERT, Staatswissenschaften, Baden-Baden 2003.

${ }^{17}$ Sidney Pollard, Free Trade, Protectionism, and the World Economy, dans: Martin GEYeR, Johannes PAULMANN (dir.), The Mechanics of Internationalism, Londres 2001, p. 27-54; Martin GEYER, One Language for the World: The Metric System, International Coinage, Gold Standard, and the Rise of Internationalism, 1850-1900, dans: ID. (dir.), Mechanics of Internationalism, p. 55-92; Madeleine HERREN, Governmental Internationalism and the Beginning of a New World Order in the Late Nineteenth-Century Europe, dans: GEYER, PAULMANN (dir.), Mechanics of Internationalism, p. 121-144.

${ }^{18}$ Heinz DUCHHARDT, Balance of Power and Pentarchie. Internationale Beziehungen 17001785. Handbuch der Geschichte der Internationalen Beziehungen, t. 4, Paderborn 1997. 
l'histoire dogmatique du droit public, de l'Empire à la Loi fondamentale ${ }^{19}$. L'ambitieuse »Histoire du droit public en Allemagne« (»Geschichte des öffentlichen Rechts in Deutschland «) de Michael Stolleis nous offre également une remarquable histoire de la science (juridique) de l'État, qui a atteint une renommée internationale. Des débuts des temps modernes à l'époque actuelle, elle tisse un lien entre l'histoire juridique dogmatique et l'histoire politique des institutions étatiques ${ }^{20}$.

Un point de fuite central des recherches historiques sur l'histoire de l'État allemand est et reste le national-socialisme, l'expérience de la ruine et de l'effondrement d'un ordre institutionnel juridique public très subtil. Aux travaux pionniers, en particulier ceux de Bernd Rüthers et de Michael Stolleis ${ }^{21}$, succédèrent bientôt de nouvelles recherches méthodologiques ${ }^{22}$, ainsi qu'un projet de recherche de l'Institut Max-Planck d'histoire du droit européen, qui replaçait l'État du national-socialisme ${ }^{23}$ dans le contexte d'un large projet comparatif à l'échelle européenne sur "L'Europe de la dictature. Contrôle économique et droit ${ }^{24}$. L'historiographie des États dictatoriaux de l'Europe du $\mathrm{XX}^{\mathrm{e}}$ siècle y est traitée dans une perspective interdisciplinaire, par des juristes, historiens et politologues, et s'appuie entre autres sur l'approche sociologique de la théorie systémique de Niklas Luhmann ${ }^{25}$. Parallèlement, d'autres recherches se font jour qui insistent plus sur les transformations structurelles des institutions et sur le caractère discriminatoire de l'État nazi, soulignant en particulier la polycratie et l'État racial ${ }^{26}$.

${ }^{19}$ Dans une brillante monographie: Christoph MölLERS, Staat als Argument, Munich 2000 (Münchener Universitätsschriften, vol. 154).

${ }^{20}$ Michael STOLLEIS, Geschichte des öffentlichen Rechts in Deutschland, 4 t. (les trois premiers tomes parus), Munich 1988-1999; traduction française du premier tome par Michel SENELLART: Histoire du droit public en Allemagne: la théorie du droit public impérial et la science de la police, 1600-1800, Paris 1998.

${ }^{21}$ Bemd RÜTHERS, Die unbegrenzte Auslegung. Zum Wandel der Privatrechtsordnung im Nationalsozialismus. Heidelberg ${ }^{5} 1997$; Michael STOLLEIS, Gemeinwohl und Minimalkonsens. Öffentliche und private Interessen in der Demokratie, dans: Aus Politik und Zeitgeschichte 28 (1978) p. 3.

${ }_{22}$ Oliver LEPSIUS, Die gegensatzaufhebende Begriffsbildung: Methodenentwicklungen in der Weimarer Republik und ihr Verhältnis zur Ideologisierung der Rechtswissenschaft im Nationalsozialismus, Munich 1994.

${ }^{23}$ Dieter GOSEWINKEL (dir.), Wirtschaftskontrolle und Recht in der nationalsozialistischen Diktatur, Francfort/M. 2005.

${ }^{24}$ Gerd BENDER, Rainer Maria KIESOW, Dieter SIMON (dir.), Das Europa der Diktatur. Steuerung, Wirtschaft, Recht, Baden-Baden 2002.

${ }^{25}$ Cf. sur cette question également la revue publiée par l'Institut Max-Planck d'histoire du droit européen "Rechtsgeschichte«; Niklas LuHMANN, Das Recht der Gesellschaft, Francfort/M. 1993.

26 Dieter REBENTISCH, Verwaltung contra Menschenführung im Staat Hitlers. Studien zum politisch-administrativen System, Göttingen 1986; Michael BURLEIGH, Wolfgang WIPPERMANN, The Racial State: Germany 1933-1945, Cambridge 1994. 
Alors que ces études se concentrent sur l'État dans son sens institutionnel, d'autres recherches s'efforcent d'appréhender l'ensemble des "systèmes d'ordre" sous l'angle d'une histoire des idées inédite et élargie. Une nouvelle collection de publications en histoire s'y consacre, dont la parution la plus récente fait ressortir combien la continuité de »la pensée de l'État« se perpétue à l'époque de l'après Seconde Guerre mondiale ${ }^{27}$.

Certaines études, qui puisent leur argumentation dans l'histoire des processus politiques et sociaux, retracent l'évolution des genres spécifiques de l'État, déconstruisant et nuançant dès lors le concept générique de l'»État«. Ces dernières jouent un rôle particulier dans l'historiographie allemande de l'État. Certaines recherches sur l'histoire de l'État de droit mettent par exemple en lumière le passage de l'État de droit formel à l'État de droit matériel ${ }^{28}$ ou encore perpétuent la tradition d'une historiographie allemande qui se concentre sur l'État sociall ${ }^{29}$. Avec la $>$ Wender de 1989, l'historiographie de la genèse et du développement de l'État-nation allemand a pris un nouvel élan. L'unification de l'Allemagne a de nouveau posé la question politique particulièrement corrosive de la spécificité nationale qu'est la formation stardive de l'Étatnation et, ce faisant, celle du "Sonderweg « allemand par rapport à l'évolution occidentale $^{30}$. Enfin, les "postcolonial studies« ont ouvert une conjoncture nouvelle aux recherches qui s'intéressaient à la constitution et aux modes de fonctionnement de l'État colonial allemand. Les travaux sur l'organisation (juridique) $^{31}$ et l'infrastructure ${ }^{32}$ de l'État colonial reposent la question de la

${ }^{27}$ Frieder GÜNTHER, Denken vom Staat her. Die bundesdeutsche Staatsrechtslehre zwischen Dezision und Integration 1949-1970, Munich 2004 (Ordnungssysteme. Studien zur Ideengeschichte der Neuzeit, vol. 15); Andreas ANTER, Die Macht der Ordnung, Tübingen 2004; Lutz RAPHAEL, Recht und Ordnung. Herrschaft durch Verwaltung im 19. Jahrhundert, Francfort/M. 2000.

${ }^{28}$ Dieter GoSEWINKEL, Adolf Amdt. Die Wiederbegründung des Rechtsstaats aus dem Geist der Sozialdemokratie (1945-1961), Bonn 1991; cf. également Edin ŚARČEVIĆ, Der Rechtsstaat, Leipzig 1996.

${ }^{29}$ Hartmut KAELBLE, Günther SCHMID (dir.), Das europäische Sozialmodell, Berlin 2004 (WZB-Jahrbuch 2004); Christoph CONRAD, Vom Greis zum Rentner. Der Strukturwandel des Alters in Deutschland zwischen 1830 und 1930, Göttingen 1994; dans une perspective française: Sandrine KOTT, L'État social allemand: représentations et pratiques, Paris, Berlin 1995 et Bénédicte ZIMMERMANN, La constitution du chômage en Allemagne: entre professions et territoires, Paris 2001.

${ }^{30}$ Heinrich August WINKLER, Der lange Weg nach Westen. 2 vol., Munich 2000; HansUlrich WEHLER, Nationalismus: Geschichte Formen Folgen, Munich ${ }^{2} 2004$; voir aussi la réédition de Theodor SCHIEDER, Nationalismus und Nationalstaat. Studien zum nationalen Problem im modernen Europa, dirigée par Otto DANN et Hans-Ulrich WEHLER, Göttingen ${ }^{2} 1992$.

${ }^{31}$ Marc GrohmanN, Exotische Verfassung. Die Kompetenzen des Reichstags für die deutschen Kolonien in Gesetzgebung und Staatsrechtswissenschaft des Kaiserreichs (18801914), Tübingen 2001; Rüdiger VOIGT, Peter SACK (dir.), Kolonialisierung des Rechts, Baden-Baden 2001; Norbert Berthold WAGNER, Die deutschen Schutzgebiete. Erwerb, Organisation, Verlust aus juristischer Sicht, Baden-Baden 2002. 
continuité de manière inédite, en analysant les rétroactions du pouvoir colonial de l'Empire allemand sur le système politique au sein de l'Empire, en particulier sur les institutions de l'État racial nazi ${ }^{33}$.

\section{Les recherches sur une institution étatique: \\ l'exemple de la citoyenneté}

Un objet de recherche, dont différentes disciplines se sont saisies dans les années 1990, relie l'historiographie du droit public, de l'État-nation et de l'État social: l'histoire de la nationalité et de la citoyenneté allemande. La nationalité en tant qu'institution juridique décidait de l'inclusion ou de l'exclusion de l'individu dans ou hors de l'État. Elle transmettait des droits civiques, politiques et sociaux fondamentaux qui donnaient la possibilité à l'individu de prendre part à la vie économique, politique et socioculturelle, et déterminaient dès lors la répartition des chances élémentaires de l'existence. Le système de droit public de l'inclusion et de l'exclusion était-il étroitement lié à des modèles spécifiquement nationaux de l'émergence de l'État-nation et du nationalisme? Au début des années 1990, certains chercheurs proposèrent une nouvelle version, plus nuancée, de la thèse du "Sonderweg" allemand, qui expliquait la politique de la nationalité et de la naturalisation, apparemment particulièrement restrictive, par un concept allemand de la nation, ethnoculturel et hostile à l'assimilation et l'opposait au modèle politique français de la nation et de la nationalité, particulièrement assimilateur ${ }^{34}$.

Mais de nouvelles recherches montrent le flou et les distorsions de cette opposition. La confrontation idéal-typique de deux modèles de l'État-nation selon les méthodes de la sociologie historique omet la contingence des configurations politiques et sociales, qui ont forgé les singularités et les radicalisations de l'État-nation ainsi que sa politique de la nationalité ${ }^{35}$. „L'État « en Allema-

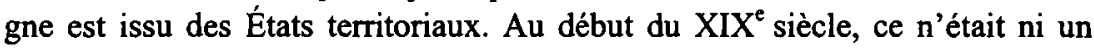

\footnotetext{
${ }^{32}$ Dirk van LAAK, Imperiale Infrastruktur, Deutsche Planungen für eine Erschließung Afrikas, 1880-1960, Paderborn 2004.

${ }^{33}$ Sur ce point, Birthe KUNDRUS, Moderne Imperialisten. Das Kaiserreich im Spiegel seiner Kolonien, Vienne 2003; ID., Von Windhoek nach Nürnberg? Koloniale »Mischehenverbote» und die nationalsozialistische Rassengesetzgebung, dans: ID. (dir.), Phantasiereiche. Zur Kulturgeschichte des deutschen Kolonialismus, Francfort/M. 2003, p. 110-134.

${ }^{34}$ Rogers BRUBAKER, Citizenship and Nationhood in France and Germany, Cambridge 1992.

${ }^{35}$ Dieter GoSEWINKEL, Einbürgern und ausschließen, Göttingen ${ }^{2} 2003$; ID., Staatsangehörigkeit in Deutschland und Frankreich im 19. und 20. Jahrhundert, dans: Christoph CONRAD, Jürgen KOCKA (dir.), Staatsbürgerschaft in Europa. Historische Erfahrungen und aktuelle Debatten, Hamburg 2001, p. 48-62.
} 
État centralisé ni national, contrairement à l'État de la Révolution française. Jusqu'en 1866, les États de la structure lâche qu'a été la Confédération germanique se sont traités réciproquement comme des pays étrangers. Ce n'est qu'après la révolution avortée de 1848 et la fondation de l'Empire allemand en 1871 qu'il devint nécessaire de donner une définition en droit public de "l'Allemand«. Il fallut attendre le dernier tiers du XIX ${ }^{\mathrm{e}}$ siècle pour que s'accomplisse un processus de snationalisation', dans lequel les critères définissant qui était et devait devenir Allemand s'uniformisèrent progressivement, se snationalisèrent, sous la pression de l'hégémonie politique prussienne. Cette situation politique et géographique particulière du stardif «État-nation - comparé aux autres grands États européens - avec ses frontières ouvertes au cœur de l'Europe, généra des mécanismes régulateurs spécifiques de la politique publique en matière de nationalité et de migration. D'une part, l'État industriel en plein essor attira énormément d'immigrés en Allemagne, issus notamment des régions pauvres d'Europe centrale et orientale, à la recherche d'un lieu de résidence sûr et d'un travail. De l'autre, l'immigration issue des régions majoritairement polonaises d'Autriche et de Russie coïncida avec les âpres luttes des nationalités que se sont livrées au sein de l'Empire allemand la majorité germanophone d'un côté, et la minorité polonaise mais aussi danoise de l'autre. Les réflexes de rejets nationaux, religieux et antisémites s'associèrent à une politique de la nationalité, au sein de laquelle la bureaucratie allemande, en majorité protestante, posa de nombreux obstacles à la naturalisation d'immigrés polonais de confession juive et catholique en particulier, mais également à celle d'autres immigrés, les politiquement indésirables par exemple.

Dans cette situation, le principe de la filiation qui régissait la nationalité changea de fonction. $\cdot \dot{A}$ l'origine introduit, sous influence française ${ }^{36}$, comme instrument de la modernisation étatique, de la détermination exacte de l'appartenance nationale à une époque de mobilisation territoriale, il se mua dans l'État-nation allemand en régulateur central de l'immigration et de la naturalisation. Mais à l'antithèse de l'idée d'une évolution linéaire de l'État-nation allemand, le principe ethnoculturel de la nationalité ne régnait pas encore avant la Première Guerre mondiale. Influencée par des conceptions de l'égalitarisme national, la loi sur l'Empire et la nationalité de 1913, qui définit la nationalité allemande tout au long du $\mathrm{XX}^{\mathfrak{e}}$ siècle, subordonnait les ambitions nationales de la "communauté ethnique " (Volksgemeinschaft) aux exigences militaronationales de la "communauté de défense« (Wehrgemeinschaft). Ce n'est qu'avec la Première Guerre mondiale et la radicalisation des représentations de la violence, du nationalisme et de l'antisémitisme que se dégagea un accès à l'adoption d'un principe "ethnique" (völkisch) et raciste de la nationalité

\footnotetext{
${ }^{36}$ Pour plus de détails dans une perspective française, se reporter à Patrick WEIL, Qu'est-ce qu'un Français?, Paris 2002.
} 
allemande. L'amputation territoriale et la discrimination nationale de l'Empire allemand, l'organisation d'un irrédentisme des Allemands de l'étranger et la gravité de la double crise politique et économique sous la République de Weimar contribuèrent massivement à ce que le national-socialisme réduise à néant deux éléments fondamentaux de >l'étaticité modernes: l'égalité constitutionnelle et l'obligation de protéger tous les membres de l'État. Les effets de radicalisation due à l'instabilité idéelle et territoriale de l'État-nation, à son hétérogénéité politico-culturelle et à ses conflits identitaires, ainsi qu'à la violence de la guerre et à la dépression économique, contribuèrent à définir les critères de l'appartenance à l'État de manière plus restrictive et plus essentialiste que ne le proposait le principe territorial de la France, politiquement plus ouvert. Avec la garantie de la stabilité des frontières territoriales de l'État-nation allemand après 1989 , le fossé entre les deux traditions stato-nationales commença à se réduire: selon le nouveau droit de la nationalité allemand entré en vigueur en 2000, l'appartenance à l'État ne découle plus uniquement de la filiation, mais aussi de la stabilité du lien avec le territoire étatique.

\section{Par-delà les frontières}

Depuis les années 1980, depuis le "cultural turn " en sciences humaines et sociales, depuis le tournant politique en Europe et l'obligation que la ,Wendes imposa de s'ouvrir aux thèmes globaux et mondiaux, les perspectives de la recherche sur l'État se sont aussi transformées. Inspiré par les méthodes de l'anthropologie historique, James Scott a proposé sa brillante analyse de l'État qui domestique et catégorise: l'État de »l'ultra modernité autoritaire ${ }^{37}$. Tout comme Scott, Dirk van Laak s'intéresse aux discours sur la planification et l'infrastructure étatique 'et met en relief les continuités des planifications infrastructurelles coloniales, des origines de la colonisation allemande jusqu'aux projets d'aide humanitaire de l'après Seconde Guerre mondiale ${ }^{38}$. Également inspirées par les méthodes de l'anthropologie historique sur les débuts des temps modernes, des recherches étudient désormais les formes et les symboles de la représentation étatique que Johannes Paulmann ${ }^{39}$, avec une méthodologie remarquable, analyse au prisme des rituels de rencontres de monarques durant

${ }^{37}$ James C. SCOTT, Seeing like a State, New Haven, Londres 1998.

${ }^{38}$ Dirk van LAAK, Imperiale Infrastruktur, Deutsche Planungen für eine Erschließung Afrikas, 1880/1960, Paderborn 2004.

${ }^{39}$ Johannes PAULMANN, Pomp und Politik. Monarchenbegegnungen in Europa zwischen Ancien Régime und Erstem Weltkrieg, Paderborn 2004. 
le slong $\mathrm{XIX} \mathrm{X}^{\mathrm{e}}$ siècle`. De nouvelles approches historiques interrogent aussi la genèse et les modes de fonctionnement de la statistique nationale ${ }^{40}$.

Outre de nouvelles méthodes, certains thèmes prétendument en marge des activités de l'État, comme par exemple le travail des services secrets ${ }^{41}$, attirent aussi la curiosité des historiens. Karl Schlögel, quant à lui, ramène la catégorie de l'espace au centre de la science historique allemande et analyse, entre autres, l'importance des conceptions spatiales et de la cartographie dans la délimitation et la politique des États-nations ${ }^{42}$.

Enfin, les travaux sur l'histoire de l'État allemand franchissent de plus en plus ses frontières et prennent une optique comparative. D'un côté, la comparaison traditionnelle entre la France et l'Allemagne se perpétue avec les recherches de Jakob Vogel sur le "culte de la nation en armes" sous l'Empire et la III république, ainsi que celles de Moritz Föllmer sur les industriels et hauts fonctionnaires en France et en Allemagne ${ }^{43}$. À la lumière d'une institution étatique commune - le service du travail obligatoire pour les hommes - Kiran Patel mène une comparaison entre la politique sociale de l'Allemagne nazie et celle des USA durant le New Deal. Dans son ensemble, l'historiographie récente de l'État tend vers la recherche de relations transnationales: vers la comparaison et le transfert d'institutions étatiques, de références théoriques et de modèles de mentalité. Cette perspective, partie de l'Europe ${ }^{44}$, s'étend progressivement à l'histoire globale ${ }^{45}$. L'État européen entendu comme une tradition institutionnelle qui, au cours de la politique coloniale du XIX ${ }^{\mathfrak{e}}$ siècle, s'imposa sur une grande partie de la planète jusqu'à une époque avancée de l'ère postcoloniale, pourrait constituer un sujet qui intégrerait les figures de l'État en Allemagne et en France, afin de les replacer par la suite dans une perspective comparative plus large.

\footnotetext{
${ }^{40}$ Adam TOOZE, Statistics and the German state, 1900/1945, Cambridge 2001.

${ }^{41}$ Christof MAUCH, Schattenkrieg gegen Hitler. Das Dritte Reich im Visier der amerikanischen Geheimdienste 1941/1945, Stuttgart 1999.

${ }^{42}$ Karl SCHLÖGEL, Im Raume lesen wir die Zeit, Munich 2003; sur la cartographie ethnographique: Morgane LABBÉ, La carte ethnographique de l'Empire autrichien: la multinationalité dans "L'Ordre des Choses", in: Comité français de cartographie 180 (2004) p. 71-84.

${ }^{43}$ Jakob VOGEL, Nationen im Gleichschritt. Der Kult der »Nation in Waffen « in Deutschland und Frankreich, 1871-1914, Göttingen 1997; Moritz FÖLLMER, Die Verteidigung der bürgerlichen Nation. Industrielle und hohe Beamte in Deutschland und Frankreich 1900-1930, Göttingen 2003.

${ }^{44}$ Pour une approche globale, cf. l'ample recherche de Wolfgang REINHARDT, Geschichte der Staatsgewalt, München ${ }^{2} 2000$. Pour une esquisse de l'évolution de la démocratie en Europe vers la démocratie européenne, cf. Hartmut KAELBLE, Wege zur Demokratie, Stuttgart, Munich 2001.

${ }_{45}$ James SCOTT, Seeing like a State; pour une ébauche programmatique: Jürgen OSTERhaMMEL, Geschichte der Globalisierung: Dimensionen, Prozesse, Epochen, Munich ${ }^{2} 2004$.
} 